Corrigendum

\title{
Corrigendum to "Effect of Transcutaneous Vagus Nerve Stimulation at Auricular Concha for Insomnia: A Randomized Clinical Trial"
}

\author{
Yue Jiao $\mathbb{D}^{1},{ }^{1}$ Xiao Guo, ${ }^{1}$ Man Luo, ${ }^{1}$ Suxia $L i,{ }^{2}$ Aihua Liu, ${ }^{3}$ Yufeng Zhao $\left(D,{ }^{4}\right.$ Bin Zhao, \\ Dequan Wang, ${ }^{2}$ Zaifang $\mathrm{Li}^{2}{ }^{2}$ Xiaojiao Zheng, ${ }^{2}$ Mozheng $\mathrm{Wu},{ }^{1}$ and Peijing Rong $\mathbb{D}^{1}$ \\ ${ }^{1}$ Institute of Acupuncture and Moxibustion, China Academy of Chinese Medical Sciences, Beijing 100700, China \\ ${ }^{2}$ National Institute on Drug Dependence, Peking University, Beijing 100191, China \\ ${ }^{3}$ Neurology Department, Xuanwu Hospital of Capital Medical University, Beijing 100053, China \\ ${ }^{4}$ Chinese Medicine Data Center, China Academy of Chinese Medical Sciences, Beijing 100700, China \\ Correspondence should be addressed to Peijing Rong; drrongpj@163.com
}

Received 12 November 2020; Accepted 12 November 2020; Published 1 December 2020

Copyright $\odot 2020$ Yue Jiao et al. This is an open access article distributed under the Creative Commons Attribution License, which permits unrestricted use, distribution, and reproduction in any medium, provided the original work is properly cited.

In the article titled "Effect of Transcutaneous Vagus Nerve Stimulation at Auricular Concha for Insomnia: A Randomized Clinical Trial” [1], there was an error in Figure 1. The label in blue is to be corrected to "tn-VNS." The authors confirm that this does not affect the results and conclusions of the article, and the corrected Figure 1 is as follows:

The authors also wish to correct the funding statement in the acknowledgements as follows:

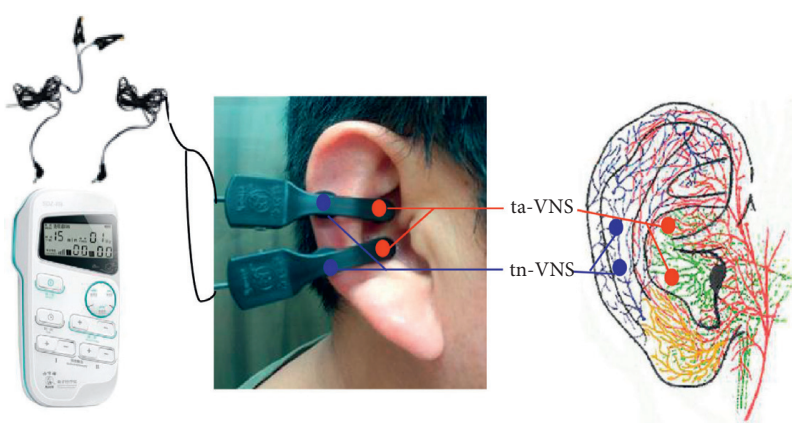

FIGURE 1: Stimulating regions of ta-VNS and tn-VNS. Red dots indicate regions of ta-VNS (ABVN: green color); blue dots indicate regions of tnVNS (LON: blue color). 


\section{Acknowledgments}

The authors would like to acknowledge and thank Yating $\mathrm{Wu}$, who assisted this study. This work was supported by the National Key Research and Development Plan (2018YFC1705800) and National Natural Science Foundation of China (81473780).

\section{References}

[1] J. Yue, X. Guo, M. Luo et al., "Effect of transcutaneous Vagus Nerve stimulation at auricular Concha for Insomnia: a randomized clinical trial," Evidence-Based Complementary and Alternative Medicine, vol. 2020, Article ID 6049891, 7 pages, 2020. 